(Aus dem anatomischen Institut zU Erlangen.)

\title{
ZUR ANATOMIE
}

DES

\section{CAVUM LARYNGIS DES MENSCHEN.}

VON

AUGUST GERLACH, ERLANGEN.

Hierzu die Tafeln $X X X / X X X V$. 
Im Gegensatz zu den meisten Hohlräumen des mensch. lichen Körpers, deren Form, Raumverhältnisse und Inhaltsgebilde durch Anwendung verschiedener Methoden der Konservierung und Präparation genauer festgestellt werden komnten, ist von seiten der Untersucher die Höhle des Kehlkopfs in Bezug auf ihre wechselnden Raum- und Gestaltverhältnisse bisher nur in geringem Grade berücksichtigt worden. Nur eine diesbezügliche Arbeit findet sich in der Litteratur ${ }^{1}$ ), die sich jedoch darauf beschränkt, die Durchmesser des unteren Kehlkopfraumes in Durchschnittszahlen wiederzugeben; die damals erhaltenen Resultate beschränken sich aber auf eine nur kleine Anzahl von Untersuchungen und beanspruchen, da sie die wichtigsten Teile, die Stimmbänder, ganz unberücksichtigt liessen, kein allgemeineres Interesse.

Auf Veranlassung und nach Angaben des Herrn Prof. Dr. L. Crerlach habe ich im anatomischen Institute zu Erlangen Untersuchungen angestellt, bei denen hauptsächlich die Altersund Geschlechtsverschiedenheiten des Kehlkopfraumes bezüglich seiner Form und Weite berücksichtigt werden sollten. Die dabei in Anwendung kommende Methode beruhte im wesentlichen darauf, dass Metallausgüsse der Kehlkopfhöhle hergestellt wurden, an denen sich das verschiedene Verhalten der einzelnen von

1) Weinberg, Über den Kehlkopf in verschiedenen Lebensaltern. Arch. f. klin. Chir. XXI, S. 412 . 
Männern und Weibern in verschiedenen Altern herrührenden Kehlköpfe durch Messung bestimmen liess. Allein nachdem etwa 25 derartige Ausgüsse fertiggestellt waren, ergab sich die Unmöglichkeit, die betreffenden Untersuchungen auszuführen und zwar aus folgenden Gründen:

Einmal stellte sich das mir zu Gebote stehende Material an frischen Kehlköpfen, das ich zum grössten Teile der Güte des Vorstandes des hiesigen pathologisch-anatomischen Institutes verdanke, als unzureichend für meine Zwecke heraus. Ich hätte, um diese $z u$ erreichen, d. h. um zu wirklich brauchbaren Durchschnittszahlen zu gelangen, weit über hundert Kehlköpfe nötig gehabt, die zu beschaffen mir erst im Laufe von Jahren möglich gewesen wäre.

Andererseits stellte es sich schon beim Vergleich der geringen Anzahl fertiger Ausgüsse heraus, dass es keine eigentliche Kadaverstellung des Kehlkopfes giebt, indem die Muskeln, von deren Kontraktionszuständen die Form des Cavum laryngis abhängig ist, in den einzelnen Fällen bezüglich ihrer postmortalen Spannungsverhältnisse sich verschieden verhielten. In dieser Beziehung zeigte sich nämlich, dass bei den einzelnen Kehlköpfen ein bestimmter Muskel sich vorzugsweise in Spannung befindet und lässt sich hierbei als wohl allgemein gültig feststellen, dass bei den mehr starren männlichen Kehlköpfen mehr die Kontraktion des M. crico-arytaenoideus lateralis vorherrscht, während bei den nachgiebigeren weiblichen Kehlköpfen der M. cricoarytaenoideus posterior die Oberhand hat.

Demgemäss ergaben sich an den Ausgüssen der männlichen und weiblichen Kehlköpfe entsprechende Ungleichheiten, die, da sie nicht auf die gleiche Ursache zurückzuführen waren, keinen Vergleich zuliessen.

Schliesslich stellten sich auch meiner Absicht, an den Aus. güssen die einzelnen Räumlichkeiten in stets gleicher Weise zu messen, unüberwindliche Schwierigkeiten entgegen: So wird der 
über der Rima vestibuli gelegene obere Kehlkopfraum hinsichtlich der Grösse seines transversalen Durchmessers bei den Ausgüssen von der mehr oder minder starken Nachgiebigkeit der Plicae ary-epiglotticae abhängig, indem das einzugiessende Metall, wenn das Gewebe der Plicae ary-epiglotticae nachgiebig ist, dieselben auseinander drängt und infolgedessen wird dann der Ausguss des oberen Keblkopfraumes breitere Dimensionen zeigen als es der Fall sein würde bei einem zweiten Kehlkopf vou gleicher Grösse, dessen Plicae ary-epiglotticae straffer und unnachgiebiger sind.

Auch die obere Grenze des oberen Kehlkopfraumes, die der durch den aditus ad laryngem gelegten Ebene entspricht, lässt sich an den verschiedenen Kehlkopfausgüssen nicht immer in g]eich präciser Weise zum Ausdruck bringen. Was den mittleren Kehlkopfraum anlangt, so ist bekanntlich die Grösse der Ventrikel sehr bedeutenden individuellen Verschiedenheiten unterworfen, sodass aus diesem Grunde schon a priori davon abgesehen werden musste, an den einzelnen Ausgüssen hinsichtlich der Volums.Ausdehnung des mittleren Kehlkopfraumes vergleichende Messungen anzustellen. Doch möchte ich hier nicht unerwähnt lassen, dass eine Gegenüberstellung der von männlichen und weiblichen Kehlköpfen gewonnenen Ausgüsse mir gezeigt hat, dass aus der Ausdehnung der Ventriculi Morgagni keineswegs auf das Geschlecht geschlossen werden darf; denn es hat sich bei gleichalterigen männlichen und weiblichen Kehlköpfen mehrfach ergeben, dass die letzteren entschieden grössere Ventrikel besassen als die ersteren.

Bei dem unteren Kehlkopfraum endlich ist zwar durch eine in der Höhe des unteren Ringknorpelrandes gelegte Ebene wie durch eine solche, welche durch die freien Ränder der Stimmbänder geht, die Abgrenzung nach oben und unten noch am ehesten möglich; doch ist gerade bei dieser Abteilung des Cavum 
laryngis die Form am gleichmässigsten ausgesprochen, sodass sie nur ein geringeres Interesse beanspruchen darf.

Infolge der erwähnten Schwierigkejten, die sich meinem ur. sprünglichen Vorhaben entgegenstellten, habe ich den Schwerpunkt meiner Arbeit darauf verlegt, die durch die Funktion bedingten Änderungen des Cavum laryngis zu studieren, indem untersucht wurde, welchen Einfluss die Kontraktion einzelner Muskeln oder mehrerer gleichzeitig wirkender Muskeln auf Form und Weite des Cavum laryngis ausübt. Die hier sich aufwerfenden Fragen haben bisher weder von anatomischer noch von physiologischer Seite eine eingehende Bearbeitung gefunden. -

Ich lasse zurächst eine eingehende Schilderung der Methode folgen, die bei der Anfertigung der Ausgüsse zur Anwendung gelangte.

Der frisch aus der Leiche genommene Kehlkopf wurde äusserlich von allem überflüssigen Gewebe befreit. Das Kehlkopfinnere wurde hierauf unter fliessendem Wasser und mit Wattebausch von Schleim und sonstigen Unreinigkeiten befreit.

Von unten her wurde alsdann in der Trachea im Bereiche der beiden ersten Trachealknorpel ein runder Kork, der genau in das Lumen der Trachea passte, bis zum unteren Ringknorpelrande eingeführt, sodass dieser fest und unbeweglich aufsass. Durch äussere Ligatur ward dann der Kork in dieser Stellung befestigt.

Zum Einspannen des so vorbereiteten Kehlkopfes wurde folgende hierzu angefertigte Vorrichtung benützt:

Zwei runde etwa $20 \mathrm{~cm}$ lange Stahlstäbe von $2 \mathrm{~mm}$ Dicke haben an ihrem unteren Ende seitliche 5 mm lange Spitzen, die mit letzterem einen fast rechten Winkel bilden.

Jeder dieser beiden Stäbe ist in seinem oberen Teile mit einer verschieblichen Hülse versehen, die durch Schraube festzustellen ist. An derselben ist ebenfalls seitlich eine Spitze an- 
gefügt, die $z u$ ihr in einem nach oben offenen spitzen Winkel angebracht ist.

Die unteren Spitzen nun wurden durch die Trachea hindurch in den Kork eingespiesst, während die oberen verschieblichen Haken in die Cartilago thyreoidea seitlich dicht unterhalb der Tubercula thyreoidea superiora eingestochen wurden: Durch beiderseitig gleichmässiges Hinaufschieben dieser beweglichen Spitzen wurde der Kehlkopf in vertikaler Richtung etwas angespannt und durch Anziehung der Schrauben in dieser Stellung erhalten. Der geschilderte Apparat gestattet, den Kehl. kopf, ohne ihn direkt zu berühren und dadurch in seiner Form durch Kompression zu alterieren, zu fassen und ihn während des Eingiessens des flüssigen Metalls in der gewünschten Stellung zu halten. -

In den Fällen, in welchen der Finfluss der Kehlkopfmuskehn auf die Form und Weite des Cavum laryngis untersucht werden sollte, wurde der Kehlkopf erst dann eingespannt, nachdem er in gleich zu erörternder Weise mit Fäden versehen worden war, durch deren Anziehung und Fixation das Spiel der Kehlkopfmuskulatur nachgeahmt werden konnte.

Die Kontraktion der Musc. crico-thyreoidei wurde nachgeahmt, indem der Schildknorpel direkt unter der Incisura thyreoidea superior mittelst eines Fadens gefasst wurde, dessen anderes Ende unmittelbar unter dem Ringknorpel mit dem in die Trachea eingeführten Korke verbunden wurde. Dadurch wurden Ring- und Schildknorpel mit ihren vorderen Seiten einander genähert, wobei die Bewegung um eine Achse erfolgte, die der Verbindungslinie zwischen beiden Articulationes cricothyreoideae entsprach.

Die Wirkung der Kontraktion der Mm. crico-arytaenoidei posteriores wurde erzielt, indem ihre Sehnen bei der Insertions. stelle am Processus muscularis unterbunden wurden; darauf wurden die beiden Fäden entsprechend der Verlaufsrichtung 
der Muskelfasern schräg nach unten medial angezogen und sodann in gespanntem Zustande an den Kork angebunden.

Die Wirkung der Mm. crico-arytaenoidei laterales wurde imitiert, indein ebenfalls ihre Sehnen mittelst Faden am Processus muscularis umschlungen wurden. Die Fäden wurden sodann zwischen Schild- und Ringknorpel nach aussen und vorne durchgezogen und parallel dem Faserverlaufe gespannt. An der Stelle, an welcher sich die beiden Fäden in der Medianlinie kreuzten, wurde an dem in die Trachea eingefügten Korke ein Häkchen angebracht, an welchem die beiden ziemlich angespannten Fäden befestigt wurden.

Die Kontraktion der Mm. arytaenoidei transversi et obliqui wurde nachgeahmt, indem an den lateralen Rändern der Aryknorpel Fäden zwischen Knorpel und genannten Muskeln durchgezogen wurden, durch welche die letzteren an genannter Stelle zusammengebunden werden konnten. Dabei war natürlich darauf zu achten, dass der Knoten in die Mitte des Muskels, d. h. gleichweit von seinem obern und untern Rande zu liegen kam. Diese Stelle bildete sonach den Angriffspunkt eines jeden der beiden Fäden, welche sodann an einem Holzstäbchen befestigt wurden, das auf der Crista des Ringknorpels, dieselbe nach oben und unten überragend, aufgelegt wurde. Durch Rotation des Stäbchens konnten die beiden Fäden medianwärts angezogen und dadurch der Zug der oben genanuten Muskeln nachgeahme werden.

Dass durch die beschriebenen Manipulationen die Muskelwirkungen leicht in etwas übertriebener Weise künstlich nachgeahmt wurden, will ich gerne zugeben. Doch dürften immerhin gerade dadurch die erhaltenen Ausgüsse charakteristischere Bilder wiedergeben.

Die den Kehlkopf haltenden beiden Stäbe stehen nach oben etwas über; hier wurde ein Querstab befestigt, der die lose nach oben gezogene Epiglottis in der Höbe festhielt. 
Die Füllung des Cavum laryngis mit flüssigem Metall wurde bei einer schrägen Stellung des vorher über Wasserdämpfen erwärmten Kehlkopfes vollzogen, in welchèr seine Vorderseite nach vorn unten gekehrt war. - Das flüssige Metall wurde aus einem mit Griff versehenen Tiegel in den Kehlkopf eingegossen und zwar wurde die Höhlung bis zu den Rändern der Plicae ary-epiglotticae gefüllt, welche in der erwähnten Schrägstellung des Kehlkopfes ungefähr in einer Horizontalebene zu liegen kommen.

Das dazu benützte Woodsche Metall ist eine Legierung von folgender Zusammensetzung:

$\begin{array}{ll}\text { Wismut } & 50,0 \\ \text { Blei } & 25,0 \\ \text { Zinn } & 12,5 \\ \text { Kadmium } & 12,5\end{array}$

und schmilzt durch Erhitzung im Wasserbade bei $70^{\circ}$ Celsius. Infolge dieses relativ niederen Schmelzpunktes wurde die Schleimhaut des ausgegossenen Kehlkopfes kaum alteriert, so dass nach Entfernung des nach der Erkaltung festgewordenen Ausgusses der Kehlkopf sich noch zu weiteren Ausgüssen verwenden liess ${ }^{1}$ ).

1) Die Herausnahme des Ausgusses aus dem Kehlkopf kann selbstredend erst erfolgen, nachdem die beiden Metallstäbe, die den Keblkopf in einer gewissen Spannung halten, entfernt sind. Die Loslösung des Metallausgusses aus dem Kehlkopf ist in dem einen Falle schwerer, im andern leichter zu bewerkstelligen. Es hängt dies wesentlich von der Ausdehnung des Appendix ventriculi laryngis ab. Reicht derselbe weiter nach oben herauf, so gelingt es nur mit Hülfe eines Häkchens, die Taschenfalte über den Ausguss des Ventrikels zurückzuschieben. Erst dann lässt sich der gesamte Ausguss nach oben zu leicht aus dem Kehlkopfinnern extrahieren. Ich habe gefunden, dass die Entfernung der späteren Ausgüsse der gleichen Kehlkopfhöhlung mühevoller ist als die der anfänglich gewonnenen, da bei den ersteren die Ausdehnung des Ventrikels mit jedem neuen Ausguss etwas zuzumehmen scheint. Es hängt dies offenbar damit zusammen, dass die Schleimhaut des Ventrikels durch die häufigen Erwärmungen nachgiebiger wird und daher das einfliessende Metall eine stärkere Ausdehnung zu Wege bringt. 
Es war sogar mehrmals möglich, von ein und demselben Kehlkopf eine grössere Anzahl von Ausgüssen zu gewinnen, an welchen die durch künstlich nachgeahmte Muskelstellungen bewirkten Verschiedenheiten sehr deutlich hervortraten.

Von den auf solchem Wege erzielten Metallmodellen, welche die innere Gestalt und Form des Cavum laryugis körperlich wiedergaben, wurden nun Gipsformen genommen, welche wiederum mit Paraffin von hohem Härtegrade ausgegossen wurden. Auf diese Weise gelang es, von einem Modell mehrere Exemplare in Paraffin zu erhalten, die in beliebigen Richtungen durchschnitten werden konnten.

Jedes einzelne Modell wurde dreimal in Paraffin nachgebildet; durch das eine ein Medianschnitt hindurchgelegt; durch das andere ein Frontalschnitt und zwar ging derselbe annähernd durch die Mitte der Pars intermembranacea rimae glottidis; durch das dritte wurden zwei Horizontalschnitte gelegt, der eine in der Höhe der Rima vestibuli, der zweite in der Höhe der Rima glottidis.

Die mittelst Säge erhaltenen Schnittflächen wurden durch Abdruck unter Zuhülfenahme eines Stempelkissens direkt aufs Papier übertragen. Diese Abdrücke wurden zur Herstellung der Zeichnungen auf Tafel XXXIV und XXXV benützt, sodass diese dadurch einen hohen Grad der Zuverlässigkeit erhalten.

Im ganzen wurden folgende rerschiedene Ausgüsse hergestellt:

1. In Neutralstellung.

2. Mit Wirkung der Mm. crico-thyreoidei.

3. Mit Wirkung der Mm. crico-arytaenoidei posteriores.

4. Mit Wirkung der Mm. crico-arytaenoidei laterales.

5. Mit Wirkung der Mm. arytaenoidei obliqui et transversi.

6. Mit kombinierter Wirkung von 2 und 3. 
7. Kombination von 2 und 4 .

8.

9.

10.

11.

12.

13.

14.

$\begin{array}{lll}2 & , & 5 . \\ 3 & , & 4 . \\ 3 & " & 5 . \\ 4 & , & 5 . \\ 2,3 & , & 4 . \\ 2,3 & , & 5 . \\ 2,4 & , & 5 .\end{array}$

Die nachfolgenden Beschreibungen stützen sich hauptsäch. lich auf die von einem weiblichen Kehlkopf von 45 Jahren gewonnenen Ausgüsse, von dem es gelang 14 verschiedenartige Ausgüsse herzustellen, da diese die allen untersuchten Kehlköpfen gemeinsamen Resultate besonders hervorstechend zeigen.

1. Verhalten des Ausgusses des Cavum laryngis, ohne dass dessen Beschaffenheit durch Nachahmung der Kontraktion der Kehlkopfmuskulatur alteriert worden wäre. (Ich werde diese Stellung, um einen kurzen Ausdruck zu haben, fernerhin als „Neutralstellung" bezeichen). (cf. die Figg. unter 1.)

Die obere Kehlkopfhöhle, Vestibulum laryngis, umfasst den Raum zwischen dem Ostium pharyngeum und den freien Rändern der Plicae ventriculares. Eine dem oberen Ostium entsprechende Grenze, ebenso eine genaue Bestimmung des Volumens ist aus früher erwähnten Gründen nicht zu erzielen.

Die vordere Fläche des Ausgusses verschmälert sich der Epiglottis entsprechend nach unten, um zwischen den vorderen Enden der Plicae ventriculares zum Punctum centrale sich zu erstrecken. Sie ist wellenförmig gekrümmt, im oberen Teil in querer Richtung konvex, in der Mitte dagegen konkav, während sie nach unten wieder stark nach vorn vorspringt. In der Mitte verläuft in vertikaler Richtung, jedoch der Konkavität genau folgend, eine dem Tuberculum epiglotticum entsprechende me- 
diane Furche, welche sich nach der oberen und unteren Abteilung zu verliert; seitlich geht dieselbe mit abgerundeten Kanten in die Seitenflächen über.

Die seitlichen Flächen sind unregelmässig vierseitig, der obere Rand senkt sich der Plica ary-epiglottica entsprechend nach hinten abwärts; die untere Grenzlinie fällt zusammen mit einer Furche, die durch die Plica ventricularis verursacht wird. Die vorderen und hinteren Ränder gehen abgerundet in die Vorder- und Hinterfläche über. Etwas unterhalb des vorderen Randes im Bereiche seiner vorderen Hälfte zeigt die äussere Fläche eine diesem parallele Wulstung. Unterhaib derselben eine breite von hinten nach vorn ziehende flache Rinne. Im hinteren Teile der Seitenfläche zeigt sich eine nahezu vertikale nur wenig nach vorn abfallende kantige Linie, welche vorn und hinten von flacheren Furchen begrenzt ist; sie entspricht dem sog. Filtrum laryngis, das bekanntlich eine zwischen den Cartilagines cuneiformes und corniculatae des Kehlkopfes verlaufende kleine Furche darstellt.

Die Beschreibung der hinteren Fläche kann erst erfolgen, nachdem auch die beiden anderen Abteilungen des Cavum laryngis erörtert sind, da dieselbe auch an unserem Ausgusse dem oberen, mittleren und unteren Kehlkopfraum gemeinsam ist und keine Merkmale zur Abgrenzung dieser Räume darbietet.

Der den mittleren Kehlkopfraum repräsentierende Abschnitt unseres Ausgusses ist nur $\mathbf{2}-\mathbf{3} \mathrm{mm}$ hoch und an den Seitenflächen des Ausgusses durch die den Plicae ventriculares und vocales entsprechenden Rinuen begrenzt. Zwischen denselben springt in den vorderen zwei Dritteln bis drei Vierteln der sagittalen Kehlkopllänge jederseits ein scharfkantiger Wulst vor, der sich nach vorn unter leichtem Ansteigen bis zum Punctum centrale erstreckt. Ich werde diesen Wulst in der Folge als „Ventrikularleiste" bezeichnen, denn derselbe stellt den Ausguss der Ventriculi laryngis dar. In ihrem mitt- 
leren Drittel zeigt sie eine nach oben gehende Verlängerung, welche den Ausguss der Appendices ventriculi vorstellt, dessen wechselnde Grössenverbältnisse ich aber unberücksichtigt lassen will.

In der linearen hinteren Fortsetzung der hinteren Ventrikularleiste ist der Ausguss eben und es lässt sich an ihm weder nach oben noch nach unten eine Grenze erkennen, durch welche die dem mittleren Kehlkopfraum entsprechende Abteilung abgegrenzt werden könnte. Der Übergang dieser planen Partie in die hintere Fläche des Ausgusses erfolgt ziemlich scharfwinkelig. Die Ventrikularleiste, welche sich an ihrem hinteren Ende stark abflacht, geht an dem Ausgusse nach oben zu in die erwähnte kantige Linie des Filtrum über. Die durch die Plica ventricularis verursachten Rinnen schneiden dagegen schärfer ein. Das Punctum centrale des Ausgusses springt stark wach vorn vor und erscheint von vorn her gesehen als die Vereinigungstelle der Ventrikularleisten, zu welchen von oben her die spitz auslaufende vordere Fläche des Vestibulum-Ausgusses hinzutritt. Es sei mir gestattet, noch einige Massangaben hier anzufügen. Der Sagittaldurchmesser des mittleren Kehlkopfraumes, am Ausgusse vom Punctum centrale bis zur hinteren Fläche gemessen, beträgt $17,4 \mathrm{~mm}$. Die sagittale Ausdehnung der Ventrikularleiste beläuft sich auf $12,8 \mathrm{~mm}$, die hinter derselben gelegene Abteilung der seitlichen Wand misst 4,6 $\mathrm{mm}$. Die an den Paraffin-Modellen unseres Kehlkopfes in oben geschilderter Weise dargestellte Rima vestibuli hat die Gestalt einer Ellipse, von welcher der hintere Teil durch eine Transversallinie abgeschnitten ist. Sie hat eine Länge von $17,0 \mathrm{~mm}$, ihre breiteste Stelle misst $8,0 \mathrm{~mm}$.

Die Rima glottidis hat auf dem in ihrer Höhe durch das Paraffin-Modell gelegten Horizontalschnitt annähernd die Gestalt eines gleichschenkeligen Dreiecks mit leichten seitlichen Ausbuch- 
tungen. Sie hat eine Länge von $16,7 \mathrm{~mm}$, ihre grösste Breite beträgt $5,5 \mathrm{~mm}$.

Der Ausguss des unteren Kehlkopfraumes, welcher nach unten durch den unteren Ringknorpelrand begrenzt wird, zeigt in seiner vom Ringknorpel umfassten Abteilung ein sich gleich bleibendes Verhalten. Der Horizontalschnitt durch das Paraffin. Modell in dieser Höhe ist oval.

Nach oben zu erscheint der Ausguss in querer Richtung komprimiert und zwar in der Weise, dass man hier nur zwei Seiten und eine hintere Fläche unterscheiden kann. Die Seitenflächen gehen nach oben zu kontinuierlich in die winkelig einschneidenden Furchen über, die von den Plicae vocales herrühren. Vorne unter dem Punctum centrale befindet sich eine ziemlich scharfe Kante, bis zu der die Seitenflächen reichen. Diese Kante geht nach unten zu allmählich in die vordere Cirkumferenz des Ringknorpelteils über. Dieser Übergang ist durch eine schwache nach vorn konvexe Wölbung gekennzeichnet, welche durch das vom Eingiessen des Metalls nach vorn gedrängte Ligamentum crico-thyreoideum sich erklärt.

Die hintere Fläche des gesamten Ausgusses ist bedeutend niedriger, wie die vordere. Als am meisten auffallendes Merkmal zeigt sich eine Wulstung innerhalb ihrer mittleren Partie. Da dieser Wulst unmittelbar über den oberen Rand der Lamina cricoidea seine Lage hat, so will ich denselben kurz als „Epikrikoidwulst " bezeichnen. Der oberhalb des Epikrikoidwulstes gelegenen Teil der hinteren Fläche erscheint in longitudinaler Richtung eingebuchtet und trägt diese Einbuchtung wesentlich zur Bildung des an ihrem unteren Ende befindlichen Epikrikoid. wulstes bei. Seitlich ist diese eingebuchtete Fläche durch scharf kantige Linien begrenzt, welche ilurerseits wieder zugleich die hinteren Ränder der Seitenflächen des bezüglichen Ausgussteiles darstellen. Der unterhalb des Epikrikoidwulstes gelegene Teil der hinteren Fläche liegt in einer nach vorn unten abfallenden 
Ebene. Er verbreitert sich allmählich nach unten zu und geht ohne scharfe Grenze in die Seitenflächen über. -- Aus der Beschaffenheit der hinteren Fläche des Ausgusses ergiebt sich, dass das Cavum laryngis an seiner hinteren Wand eine Ausbuchtung besitzt, deren stärkste Vertiefung unmittelbar über den oberen Rand der Platte des Ringknorpels zu liegen kommt. Diese dürfte wohl geeigneterweise als „Epikrikoidbucht“ zu bebenennen sein.

Schliesslich möchte ich auch noch kurz der Beschaffenheit des gesamten Ausgusses gedenken, wie sie sich bei Betrachtung von vorneher ergiebt. Von vorne her gesehen, fällt vor allem eine vorspringende und kantige Pyramide auf, deren Spitze dem Punctum centrale entspricht. Die seitlichen Kanten werden von den im Punctum centrale zusammenfliessenden Ventrikularleisten gebildet; sie verlaufen horizontal, während die von oben und, unten her gehenden Kanten vertikal gestellt sind. Erstere geht vom Ausguss des Vestibulum an und ist nichts anderes als das schmal auslaufende untere Ende von dessen vorderer Fläche. Die von unten her zum Punctum centrale aufsteigende Kante gehört dem Ausguss des unteren Kehlkopfraumes an und zwar der oberhalb des Ringknorpels gelegenen Abteilung des. selben. Die Kante trennt, wie bereits erwähnt, die an diesem Abschnitt des Ausgusses vorkommenden Seitenflächen. Hierbei möchte ich nicht unerwähnt lassen, dass bei mauchen Kehlkopfausgüssen statt der vierseitigen Pyramide mehr eine dreiseitige zu sehen war, was darin seinen Grund hatte, dass die beiden Plicae vocales an ihrem vorderen Ende eine kurze Strecke mit einander verwachsen waren. Infolgedessen erschien die erwähnte vordere Kante des Ausgusses des unteren Kehlkopfraumes winkelig gebrochen und zwar nahezu unter einem rechten Winkel. Durch diesen nach rückwärts gerichteten Winkeleinschnitt teilt sich die erwähnte Kante in einen nach vorn nur sanft ansteigenden, nahezu horizontal liegenden Schenkel, welcher in dem 
Punctum centrale ausläuft, und einen unteren vertikalen Abschnitt.

Ich habe absichtlich bei der Erörterung dieses die Neutralstellung repräsentierenden Ausgusses länger verweilt, weil derselbe ein geeignetes Vergleichsobjekt bildet gegenüber den Ausgüssen, welche ich erhielt, nachdem ich vorher die Wirkung einzelner Muskeln oder die kombinierte Wirkung mehrerer Muskeln in der eingangs beschriebenen Weise nachgeahmt hatte.

2. Verhalten des Ausgusses des durch Kontraktion der Mm. cricothyreoidei veränderten Cavum laryngis (cf. Fig. 2).

Die vordere Fläche des Ausgusses vom Vestibulum laryngis erscheint ebener als in der Neutralstellung. Die vom Tuberculum epiglotticum herrührende Rinne ist scharfkantiger nach den Seitenflächen abgegrenzt.

Die Seitenflächen sind schräger gestellt, d. h. sie konvergieren nach unten stärker.

Der Ausguss des mittleren Kehlkopfraumes ist verschmälert, indem durch die stärker einspringenden Plicae ventriculares und vocales die denselben entsprechenden Rinnen des Ausgusses vertieft erscheinen. Die sagittale Länge des mittleren Kehlkopfraumes am Ausguss gemessen ist vergrössert und beträgt $19,2 \mathrm{~mm}$. Die Ventrikularleisten verlaufen nicht mehr nach vorn ansteigend, sondern ziemlich in horizontaler Richtung. Die sagittale Länge der Ventrikularleiste ist 14,9 $\mathrm{mm}$; der dahinter liegende Teil des Ausgusses misst 4,3 mm. Daraus ist ersichtlich, dass sich untér dem Einfluss der Mm. cricothyreoidei die Pars intermembranacea rimae glottidis verlängert hat, während die Pars intercartilaginea sich verkürzt hat.

Die Rima vestibuli hat ihre Form auf dem Horizontalschnitt ziemlich beibehalten, erscheint jedoch etwas in die Länge gezogen $(18,8 \mathrm{~mm})$; dementsprechend misst sie an ihrer breitesten Stelle, die sich gegen die Neutralstellung etwas nach rorn verschoben hat, nur $6 \mathrm{~mm}$. 
Die Rima glottidis zeigt gegenüber der Neutralstellung eine bedeutende Verschmälerung, ist dagegen in sagittaler Richtung mehr ausgedehnt. Ihre frontal breiteste Stelle misst nur $3,5 \mathrm{~mm}$, ihre Länge beträgt $18,0 \mathrm{~mm}$. Durch die Verschmälerung wird selbstredend eine Verkleinerung des vorderen Winkels der Rima glottidis herbeigeführt. - Die von den Plicae vocales herrührenden Rinnen schmeiden viel schärfer ein, als es in der Neutralstellung der Fall ist. Es bilden die Ventrikularleisten mit den Seitenflächen des unteren Ausgussteiles so ziemlich einen rechten Winkel.

Was den Ausguss des unteren Kehlkopfraumes anlangt, so zeigt dessen vordere Fläche eine Abflachung. Der bei der Neutralstellung hervortretende schwache Wulst fehlt. Dagegen ist das untere Ende der vorderen Fläche etwas breiter. Die Seitenflächen konvergieren infolge der stärkeren Einsenkung der von den Plicae vocales bedingten Furchen nach oben etwas stärker.

Die hintere Fläche des Gesamtausgusses ist in ihrer dem Vestibulum entsprechenden Abteilung schmäler geworden. Der Epikrikoid-Wulst ist stärker ausgebildet und springt nach hinten sehr merklich vor: Die Epikrikoidbucht ist demnach stark vertieft.

An der Vorderseite des Gesamtausgusses springt die vierseitige Pyramide des Punctum centrale spitzer vor, hat sich demnach unter Verschmälerung nach vorne verlängert.

Das Gesamtvolumen des Ausgusses ist verkleinert.

3. Verhalten des Ausgusses des durch Kontraktion der Mm. crico-arytaenoidei posteriores veränderten Cavum laryngis. (cf. Fig. 3).

Der Ausguss der oberen Kehlkopfhöhle ist im allgemeinen breiter geworden, jedoch bezüglich seiner Vorderfläche und der Seitenflächen der Neutralstellung gleich geblieben mit der Einschränkung, dass die letzteren nicht so stark nach unten konvergiereu. 
Der Ausguss des mittleren Kehlkopfraumes ist breiter geworden, was durch grösseren Abstand der beiden Ventrikularleisten seinen Ausdruck findet. Letztere sind nach vorne leicht ansteigend und nach hinten oben deutlicher zur Kante des Filtrums geschweift. Die sagittale Länge des mittleren Kehlkopfraumes am Ausguss hat um ein Geringes zugenommen und beträgt 17,6 mm. - Die Länge der Ventrikularleisten ist wegen des im ganzen breiter gewordenen Raumes auf $12,7 \mathrm{~mm}$ verkürzt; der hinter ihnen gelegene Teil misst 4,9 mm.

Die Rima restibuli ist stark erweitert; ihre breiteste Stelle liegt etwa an der Kante des Filtrums und misst 11,2 mm. Ihre sagittale Länge hat nur wenig zugenommen und misst 17,4 $\mathrm{mm}$. Die Form der Rima vestibuli auf dem Horizontalschnitt ist etwas verändert: Der grossen Breite entsprechend ist sie hinten mehr abgerundet und die vordere Spitze ist verschwunden.

Die Rima glottidis hat zungenförmige Gestalt, hinten ist sie fast halbkreisförmig begrenzt, vorne verjüngt sie sich zu einer Spitze. Der vordere Winkel ist selbstredend durch die Verbreiterung der Glottis vergrössert. Die frontal breiteste Stelle misst 9,6 mm, die sagittale Länge hat abgenommen und misst 16,4. $\mathrm{mm}$.

Der Ausguss der unteren Kehlkopfhöhle zeigt sich eben. falls verbreitert. Vom Punctum centrale siebt man zwei ganz kurze, sich bald verlierende, lineare Erhebungen schräg nach hinten und unten verlaufen, die der Ausdruck schwacher Rinnen sind, welche bei Kontraktion der Mm. crico-arytaenoidei posteriores an der betreffenden Stelle der Kehlkopfwand auftreten.

Die vordere Fläche des Ausgusses des unteren Kehlkopfraumes ist sonst eben. Die Seitenflächen stehen fast senkrecht und zwar in der Weise, dass sie hinten einander parallel sind, während sie nach vorne zu konvergieren. - Die hintere Fläche des Gesamtausgusses ist schon oben sehr breit und nimmt 
nach unten nur wenig an Breite zu. Der Epikrikoidwulst ist nur als ganz leichte Erhebung sichtbar.

Von vorne her gewahrt man am Gesamtausguss, dass die Pyramide des Punctum centrale nach allen Seiten verbreitert, jedoch auch flacher geworden ist.

Das Volumen des Gesamtausgusses der Kehlkopfhöhlung ist wesentlich vergrössert.

4. Verhalten des Ausgusses das durch Kontraktion der Mm. crico-arytaenoidei laterales veränderten Cavum laryngis. (cf. Fig. 4).

Der Ausguss des oberen Kehlkopfraumes entspricht dem bei Wirkung der Mm. crico-thyreoidei beschriebenen, nur dass vielleicht die vordere Fläche noch etwas mehr vertikal gestellt ist und die Seitenflächen nach unten zu um ein Geringes konvergenter gestellt sind. Das untere Ende ist verengt, da die von den Plicae ventriculares herrührenden Furchen sehr nahe einander liegen.

An dem Ausguss der mittleren Kehlkopfhöhle springen die Ventrikularleisten gegenüber den Seitenflächen der unteren Ausgusshälfte stärker vor, was durch die ebenfalls sehr tief einschneidenden Furchen der Plicae vocales bedingt ist. Die sagittale Länge des Raumes am Ausguss gemessen ist auf $18,1 \mathrm{~mm}$ gewachsen. Diese Verlängerung kommt hauptsächlich dem Gebiet der Ventrikularleisten mit $14,5 \mathrm{~mm} \mathrm{zu}$, während der hinter letzteren gelegene Teil auf $3,6 \mathrm{~mm}$ zurückgegangen ist.

Die Rima vestibuli auf dem Horizontalschnitt ist ebenfalls zungenförmig und zeigt in der Mitte jederseits leichte Ausschweifungen. Ihre frontal breiteste Stelle erstreckt sich über das ganze mittlere Drittel und misst $5,6 \mathrm{~mm}$; ihre sagittale Länge beträgt $17,6 \mathrm{~mm}$.

Die Rima glottidis hat auf den Horizontalschnitt durch das Paraffinmodell eine ganz eigenartige Gestaltung. Die Tremung in Pars intermembranacea und Pars intercartilaginea ist bei ihr deutlich ausgesprochen. Die Pars intermembranacea ist stark 
verschmälert, nach vorn und hinten $\mathrm{zu}$ sich verjüngend; an ihrem hinteren Ende, das den Übergang zur Pars intercartilaginea bildet, misst sie nur 1,8 mm; dann schwillt plötzlich die Pars intercartilaginea stark an $\mathrm{zu}$ einem rundlichen Lumen von $3,9 \mathrm{~mm}$ frontaler Breite. Die sagittale Länge der ganzen Rima glottidis ist vermehrt und misst $17,3 \mathrm{~mm}$. Im Ausgusse des unteren Kehlkopfraumes erscheint die Vorderfläche ganz eben und geht in eine kurze fast vertikal verlaufende, an Punctum central endigende Kante über. Die Seitenflächen konvergieren sehr stark zur Furche der Plicae vocales.

Die hintere Fläche des ganzen Ausgusses erscheint verschmälert, besonders im oberen über dem Fpikrikoid-Wulst gelegenen 'I'eil, der etwas verlängert erscheint. Der EpikrikoidWulst springt ziemlich stark vor, jedoch nicht ganz so spitz wie bei Wirkung der Mm. crico-thyreoidei.

Die Pyramide der Vorderfläche des Gesamtausgusses erscheint verschmälert, weil die Ventrikularleisten einander stark genähert sind.

Das Gesamtvolumen des Ausgusses ist verkleinert.

5. Verhalten des Ausgusses des durch Kontraktion der Mm. arytaenoidei obliqui et transversi veränderten Cavum laryngis. (cf. Fig. 5).

Der Ausguss des oberen Kehlkopfraumes ist durch das gänzliche Verschwinden der Incisura interarytaenoidea im sagittalen Durchmesser verkleinert, hat aber an Höhe zugenommen. Die vordere Fläche ist wieder wellenförmig gekrümmt, jedoch ist die Medianfurche in der Konkavität mehr ausgesprochen. Die Seitenflächen zeigen, besonders im hinteren T'eil, stärkere Konvexität und stossen mit einer hinteren Mediankante zusammen, während sie gegen die vordere Fläche eine stärker ausgesprochene Abgrenzung zeigen.

Am Ausguss des mittleren Kehlkopfraumes verlaufen die Ventrikularleisten horizontal, jedoch sind sie schon frühzeitig 
nach hinten aufwärts zur Kante des Filtrums geschweift und stehen weit von einander ab.

Es sei hier nebenbei bemerkt, dass die Appendices ventriculorum sehr stark ausgeprägt sind; dies ist wohl vor allem der mehr aufgehobenen Spannung der Ligamenta ventricularia zuzuschreiben.

Die sagittale Länge des Mittelraumes am Ausguss beträgt $17,0 \mathrm{~mm}$, ist also verkürzt. Die Ventrikularleiste ist auf $13,4 \mathrm{~mm}$ verlängert, während die binter ihr gelegene plane Fläche auf $3,6 \mathrm{~mm}$ verkürzt ist.

Die Rima vestibuli hat die Form einer Ellipse, ist am hinteren Ende abgerundet, am vorderen leicht zugespitzt. Ihre sagittale Länge beträgt nur $16,5 \mathrm{~mm}$; ihre frontal breiteste Stelle liegt fast in der Mitte und misst 7,9 mm.

Die Rima glottidis ist von breit spindelförmiger Gestalt und ist vorn und hinten mit Spitze versehen. Ihre sagittale Länge ist $16,5 \mathrm{~mm}$, die frontal breiteste Stelle misst $7,1 \mathrm{~mm}$. Die Pars intermembranacea ist erweitert, die Pars intercartilaginea dagegen hinten verschmälert.

Der Ausguss der unteren Kehlkopfhöhle zeigt an seiner vorderen Fläche die leichte Wulstung wie bei der Neutralstellung, nur in etwas geringerem Grade ausgesprochen. Die seitlichen Flächen konvergieren nach oben weniger als in der Neutralstellung und erscheinen ebenfalls etwas stärker gewölbt.

Die hintere Fläche des Gesamtausgusses zeigt im oberen und mittleren Kehlkopfraum keine Ebene, sondern nur eine Kante; neben dieser sind longitudinale Rinnen, die von der Faltung der Membrana mucosa zwischen den Aryknorpeln herrühren. Der Epikrikoidwulst tritt ziemlieh spitz vor, was dadurch verursacht wird, dass die unter ihm gelegene Fläche etwas schräg nach vorn abfällt.

Die Pyramide des Punctum centrale erscheint in querer Richtung verbreitert. 
Das Gesamtvolumen des Ausgusses ist etwas vermehrt.

Um eine Übersicht über die Verschiedenheiten, welche die sub $2-5$ beschriebenen Kehlkopfausgüsse gegenüber dem in der Neutralstellung gewonnenen Ausgusse darbieten, möglichst zu erleichtern, habe ich in der folgenden kleinen Tabelle das Verhalten der Rima glottidis bezüglich ihrer gesamten Länge und Breite, sowie der Länge ihrer beiden Abteilungen im Vergleich zur Neutralstellung zusammengestellt. Ebenso wurde in einer vierten Rubrik die grössere oder geringere Ausdehnung des Gesamtvolumens berücksichtigt.

Vergleicht man jedoch die Ausgüsse 2--5 untereinander, so ergeben sich die folgenden Abstraktionen:

Durch die Kontraktion der Mm. crico-aryt. laterales wird der mittlere Kehlkopfraum am meisten verschmälert, wobei die ventriculi laryngis sehr nahe an die Medianebene heranrücken. Das gleiche, jedoch in minder hohem Grade, ist bei der Kontraktion der Mm. crico-thyreoidei der Fall. In beiden Fällen geht die Verschmälerung mit einer Zunahme der sagittalen Durchmesser eimher. Aus der Verschmälerung des mittleren Kehlkopfraumes leitet sich eine stärkere Abnahme der Breitendimensionen der angrenzenden Abschnitte des Cavum laryngis superius und inferius ab. In beiden Fällen ist die Epikrikoidbucht ansehnlich entwickelt und zwar ist sie stärker bei Kontraktion der Mm. cricothyr. als bei der der laterales ausgebildet.

Der Einfiuss der Kontraktion der Mm. crico-aryt. posteriores sowie der $\mathrm{Mm}$. crico-aryt. obliqui und transversi, welcher an Nr. 3 und 5 ersichtlich ist, äussert sich am auffälligsten in einer starken frontalen Verbreiterung des mittleren Kehlkopfraumes. Die Ventrikel werden stark lateral geschoben. Die Verbreiterung ist jedoch eine grössere bei Kontraktion der erstgenannten Muskeln als bei der der letztgenannten. Im sagittalen Durchmesser erscheint in beiden Fällen der mittlere Kehlkopfraum reduziert. Durch die Wirkung der die beiden Ary- 

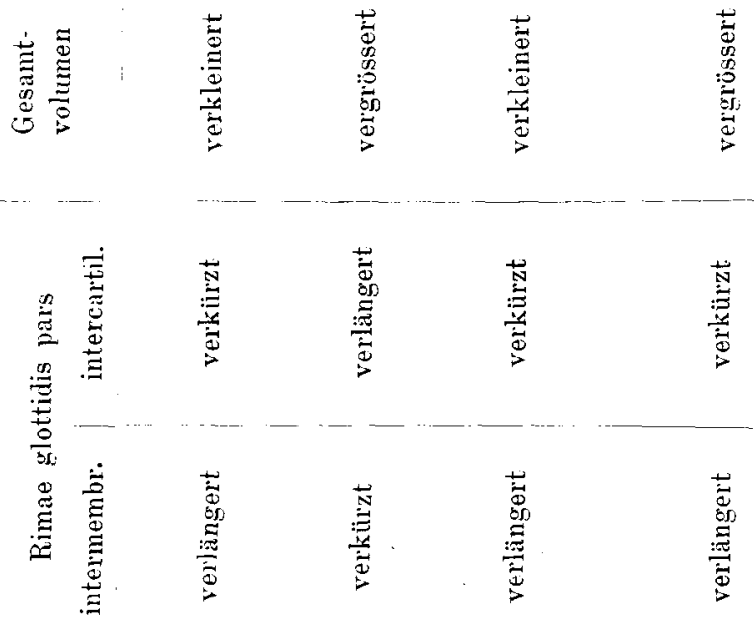

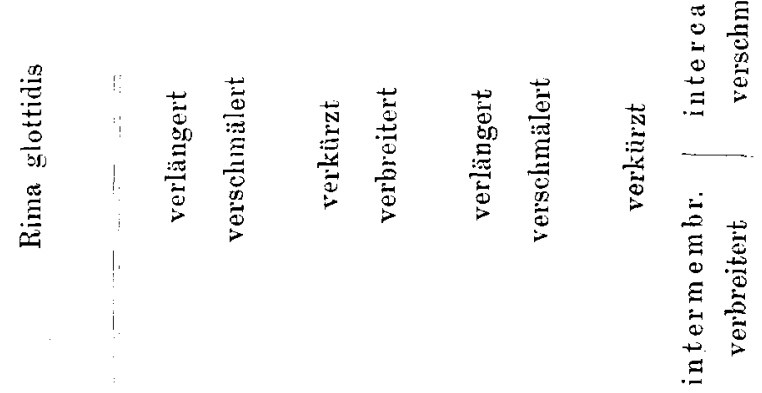

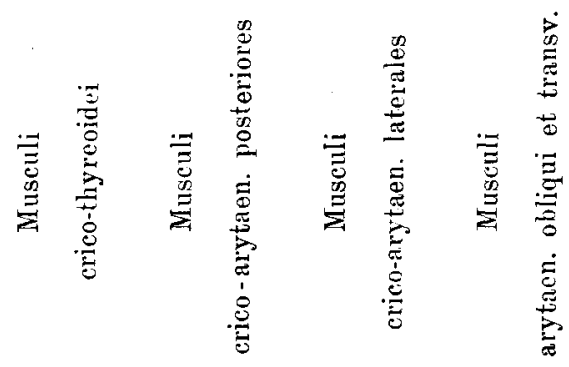


knorpel verbindenden Muskeln erleidet der mittlere Kehlkopfraum in seinem hinteren Teile neben der Verkürzung in der Sagittalen zugleich eine Einengung, während er hier bei kontrahierten Mm. crico-aryt. posteriores sehr breit erscheint. Damit steht in Zusammenhang, dass im ersteren Falle die Epikrikoidbucht sehr stark in Erscheinung treten muss, während sie sich im zweiten bedeutend abflachen muss.

Ich lasse jetzt die Beschreibung der Ausgüsse folgen, wie sie nach imitierter Wirkung von 2 resp. 3 gleichzeitig kontrahierten Muskeln erhalten wurden.

6. Verhalten des Ausgusses des durch Kontraktion der Mm. crico-thyreoidei (2) und crico-arytaen. posteriores (3) veränderten Cavum laryngis. (cf. Fig. 6.)

Der Ausguss der oberen Kehlkopfhöhle gleicht im ganzen dem Ausguss Nr. 3, nur ist die Verschmälerung nach unten infolge der Wirkung der Mm. cricothyreoidei stärker ausgesprochen.

Im mittleren Kehlkopfraume fallen die Ventrikularleisten entschieden etwas nach vorne ab. Ich vermute als ursächliches Moment dieser Erscheinung ein durch die Kontraktion der $\mathrm{Mm}$. crico-arytaen. posteriores bedingtes Emporsteigen der Processus vocales und damit zugleich auch der hinteren Enden der Plicae vocales. - Weniger scharf markiert ist die nach vorn abfallende Stellung der Ventrikularleisten übrigens auch schon bei Ausguss 3 erkenntlich (alleinige Wirkung des M. crico-aryt. posterior).

Die sagittale Länge des mittleren Kehlkopfraumes ist 18,6 mm, hält sich demnach auch so ziemlich in der Mitte zwischen den bezüglichen Maassen von Ausguss 2 und 3. Die Ventrikularleisten sind länger $(15,8 \mathrm{~mm})$ als bei 2 und 3 , die hinter ihnen gelegenen Teile dagegen etwas kürzer $(2,8 \mathrm{~m})$.

Die sagittale Länge der Rima vestibuli misst $18,2 \mathrm{~mm}$, ist also länger als bei 3 und kürzer als bei 2 ; sie steht auch bezüglich ibrer Breite mit 9,5 mm zwischen beiden in der Mitte, 
diese breiteste Stelle liegt etwa an der Filtrum-Kante. Ihr Horizontalschnitt entspricht in hinteren Teil mehr der Beschaffenheit von 3 , im vorderen mehr von 2, worin ebenfalls die Wirkung beider Muskeln zum Ausdruck kommt.

Die Rima glottidis verhält sich ganz ähnlich; ihre Länge ist $17,5 \mathrm{~mm}$; ihre Breite beträgt $8,9 \mathrm{~mm}$.

Auch das Cavum inferius stimmt vorn mehr mit 2, hinten mehr mit 3 überein.

Der Epikrikoidwulst zeigt ebenfalls eine zwischen 2 und 3 in der Mitte stehende Ausbildung.

Aus den angeführten Befunden ergiebt sich, dass die Kon traktion der Mm. crico-thyreoidei mehr die vordere Hälfte, die der Mm. crico-arytaen. posteriores mehr die hintere Hälfte des Cavum laryngis beeinflusst.

7. Verhalten des Ausgusses des durch Kontraktion der Mr. crico-thyreoidei (2) und crico-aryt. laterales (4) veränderten Cavum laryngis. (cf. Fig. 7).

Am Ausguss der oberen Kehlkopfhöhle fällt eine ungemein stark nach unten zu gerichtete Verschmälerung auf, welche sich bedeutender als bei 2 und 4 darstellt.

Die Ventrikularleisten stehen nahezu etwas horizontal, eher etwas nach vorn abwärts geneigt. Ihre sagittale Länge beträgt $16,9 \mathrm{~mm}$, der hinter ihnen gelegene Teil misst $4,4 \mathrm{~mm}$, die Länge des ganzen Mittelraumes der Kehlkopfhöhle ist 21,3 mm. Alle diese Verlängerungen sind durch summierte Wirkung der beiden Muskeln entstanden.

Die Rima vestibuli erscheint auf dem Horizontalschnitt fast überall gleich breit. Ihre Länge misst $20,5 \mathrm{~mm}$, ihre breiteste Stelle $=4,2 \mathrm{~mm}$.

Die Rima glottidis zeigt sich auf $19,3 \mathrm{~mm}$ verlängert und ist sehr schmal. Ihre breiteste Stelle liegt etwa $1,0 \mathrm{~mm}$ vor der hinteren Begrenzungslinie und misst $2,2 \mathrm{~mm}$. Sie hat auf 
dem Horizontalschnitt lanzettförmige Gestalt, geht aber vorn nicht ganz spitz zu.

An der hinteren Wand kommt der Epikrikoidwulst ausnehmend stark zum Ausdruck, was sich dadurch erklüren dürfte, dass mit der sehr starken Verengerung der Pars intercartilaginea eine Verlängerung derselben nach hinten einhergeht, welche unmittelbar über der Platte des Ringknorpels am stärksten zum Ausdruck kommt. Ausserdem zeigt sich an der vorderen Wand des Ausgusses eine starke Einbiegung, die durch die nach abwärts gerichtete Lageveränderung des Schildknorpels verursacht sein dürfte.

Die Pyramide des Punctum centrale ist sehr schmal, in die Länge gezogen und mehr dreikantig, indem die untere Kante noch stärker als bei Ausguss 2 beinahe horizontal eine kurze Strecke lang mach rückwärts verläuft, um erst dann eine mehr vertikal nach hinten abfallende Richtung anzunehmen. Auch diese Erscheinung muss dem Muskelzug der M. crico-thyr. zugeschrieben werden, bei dem sich das Punctum centrale nach vorne zu (und abwärts) vorschiebt, wobei es zu einer teilweisen Aneinanderlagerung der vorderen Enden der Plicae vocales kommt.

Das Volumen des Gesamtausgusses ist erheblich verringert.

Durch diesen Ausguss wird die im gleichen Sinne erfolgende Wirkung der beiden beteiligten Muskeln in augenfälliger Weise illustriert, welche auf einer maximalen Verengerung der Rima glottidis beruht. - Ein völliger Schluss der Rima glottidis scheint jedoch durch die beiden Muskeln allein nicht zu Wege gebracht $\mathrm{zu}$ werden. Ich werde hierauf noch später zu sprechen kommen und zwar bei Ausguss 14, der dadurch erhalten wurde, dass ausser den zwei in Rede stehenden Muskeln auch noch die $\mathrm{Mm}$. arytaenoidei transversi et obliqui berücksichtigt wurden. Doch sei hier schon erwähnt, dass sich meinen Untersuchungen 
nach auch durch das Hinzutreten letztgenannter Muskeln kein vollständiger Schluss der Rima glottidis erreichen liess.

8. Verhalten des Ausgusses des durch Kontraktion der Mm. crico-thyreoidei (2) und arytaenoidei obliqui et transversi (5) veränderten Cavum laryngis. (cf. Fig. 8).

Der Ausguss des oberen Kehlkopfraumes entspricht in seiner Gestalt Nr. 5, nur dass er in seinem unteren Teil etwas schmäler erscheint, was auf Rechnung der gleichzeitig wirkenden $\mathrm{Mm}$. crico-thyreoidei zu setzen ist.

In der mittleren Kehlkopfhölle stehen die Ventrikularleisten horizontal, ihre Länge beträgt $16,1 \mathrm{~mm}$, während der hinter ihnen gelegenen Teil 2,5 $\mathrm{mm}$ misst. Die sagittale Länge des ganzen Raumes misst daher $18,6 \mathrm{~mm}$.

Die Rima vestibuli entspricht auf dem Horizontalschnitte dem Durchschnitt einer Konvexlinse, jedoch ist die vordere Spitze schärfer als die hintere, die mehr abgerundet ist. Die sagittale Länge misst 18,1 , die breiteste Stelle $6,6 \mathrm{~mm}$.

Die Rima glottidis ähnelt der von Ausguss 2, ist aber mit $17,5 \mathrm{~mm}$ etwas kürzer als bei Nr. 2 ; ilıre grösste Breite beträgt $3,9 \mathrm{~mm}$.

Gestalt und Dimensionen des Cavum inferius gleichen mehr Nr. 5, nur ist durch Wirkung der Mm. crico-thyreoidei eine stärkere Verschmälerung bedingt worden.

Der Epikrikoidwulst zeigt die gleiche Ausbildung wie bei Nr. 5.

Die Pyramide des Punctum centrale ist durch Wirkung der Mm. crico-thyreoidei in die Länge gezogen.

Es zeigt dieser Ausguss, wie a priori zu erwarten war, dass die Musc. arytaen. obliqui et transversi mehr gestaltverändernd auf die hintere Hälfte der Kehlkopfhöhle einwirken; doch geht ferner aus der Form des Ausgusses hervor, dass bezüglich der Verengerung der Rima glottidis die Wirkungen der Mm. cricothyreoidei bei gleichzeitiger Kontraktion der Mm. arytaen. obli- 
qui et transversi nicht so zur Geltung kommen können als bei dessen alleiniger Kontraktion.

9. Verhalten des Ausgusses des durch Kontraktion der Mm. crico-arytaen. posteriores (3) und crico-aryt. laterales (4) veränderten Cavum laryngis. (cf. Fig. 9).

Der Ausguss der oberen Kehlkopfhöhlung entspricht dem Nr. 3, jedoch ist die Vorderwand weniger gekrümmt.

Im Cavum medium sind die Ventrikularleisten infolge Emporrückens der Proc. vocales wenig nach vorn abfallend; sie erscheinen länger als bei den früher beschriebenen Ausgüssen und messen $17,0 \mathrm{~mm}$, die hinter ihnen liegenden Teile sind 3,5 mm lang. Der Gesamtraum ist in seiner sagittalen Länge auf $20,5 \mathrm{~mm}$ gewachsen.

Die Gestalt der Rima vestibuli gleicht einem gleichschenkligen Dreieck mit rückwärts gestellter Basis, dessen hintere Winkel abgerundet erscheinen und dessen Seitenlinien leicht lateral ausgeschweift sind. Ihre sagittale Länge $=19,9$, die grösste Breite misst 10,8 mm.

Die Gestalt der Rima glottidis ist ähnlich der der Rima vestibuli, nur ist der vordere Winkel spitzer und die beiden hinteren Winkel sind mehr abgerundet. Ihre sagittale Länge misst $19,1 \mathrm{~mm}$, die breiteste Stelle $9,1 \mathrm{~mm}$.

Im Cavum inferius ist der Epikrikoidwulst unansehnlich, jedoch stärker als bei Wirkung der Mm. crico-aryt. posteriores allein.

Die Pyramide verhält sich wie bei Nr. 3 .

Das Gesamtvolumen des Ausgusses ist stark vermehrt.

Der Ausguss zeigt, dass die besonders den mittleren Kehlkopfraum betreffenden Dilatationen des Cavum laryngis, die die $\mathrm{Mm}$. crico-aryt. posteriores ausüben, durch die gleichzeitige Wirkung der Mm. crico-aryt. laterales etwas abgeschwächt werden. Selbstverständlich beeinflussen die beiden Muskeln hauptsächlich 
den hinteren Teil der Kehlkopfhöhle, während der vordere Teil in weit geringerem Grade in Mitleidenschaft gezogen wird.

10. Verhalten des Ausgusses des durch Kontraktion der $\mathrm{Mm}$. crico-arytaen. posteriores (3) und arytaen. obliqui et transversi (5) veränderten Cavum laryngis. (cf. Fig. 10).

Der Ausguss des Vestibulum laryngis entspricht dem bei Nr. 3 mit dem Unterschied, dass eine Verschmälerung in ge. geringem Grade an seinem vorderen, stärker an seinem hinteren Teil ausgeprägt ist.

Die Ventrikularleisten sind leicht nach vorne abfallend, hinten stark aufwärts geschweift. Sie sind ebenso wie der hintere plane Teil verkürzt auf $15,2 \mathrm{~mm}$ bezw. $1,6 \mathrm{~mm}$. Die Länge des ganzen Mittelraumes ist $=16,8 \mathrm{~mm}$.

Die Rima vestibuli hat nahezu die Gestalt eines kurzen Ovals, das jedoch vorne zugespitzt ist. Die sagittale Länge misst $16,1 \mathrm{~mm}$, die grösste Breite beträgt $11,1 \mathrm{~mm}$.

Die Rima glottidis hat ähnliche Gestalt, erscheint aber kürzer $(17,0 \mathrm{~mm})$ und breiter $(10,5 \mathrm{~mm})$.

Der Epikrikoidwulst ist verbreitert und verflacht.

Der Pyramide des Punctum centrale entspricht der bei Nr. 5.

Das Volumen des ganzen Ausgusses ist vermehrt.

Die geschilderten Form- und Grössenverhältnisse geben ein äusserst anschauliches Bild hinsichtlich der kombinierten Wirkung der beiden beteiligten Muskeln. Die Länge der Rima vestibuli mit $16,1 \mathrm{~mm}$ ist geringer als das entsprechende Mass sämtlicher anderen Kehlkopfausgüsse, während ihre Breite allerdings nicht so gross ist als bei alleiniger Wirkung der Mm. crico-aryt. posteriores.

Was die Rima glottidis anlangt, so hat sich mir eine geringere Länge (als $17,0 \mathrm{~mm}$ ) nur bei alleiniger Wirkung der $\mathrm{Mm}$. crico-aryt. posteriores ergeben $(16,4 \mathrm{~m})$; dagegen ist ihre Breite $(10,5 \mathrm{~mm})$ eine so ausserordentliche, dass sie sich bei 
keinem anderen Ausguss findet. Daraus geht hervor, dass die kombinierte Wirkung der beiden in Rede stehenden Muskeln in einer maximalen Verbreiterung der Rima glottidis gipfelt, die natürlich mit einer ansehnlichen Verkürzung verbunden sein muss.

11. Verhalten des Ausgusses des durch Kontraktion der Mm. crico-aryt. laterales (4) und aryt. transv. et obliqui (5) veränderten Cavum laryngis. (cf. Fig. 11).

Der Ausguss der oberen Kehlkopfhöhle erscheint schmal und nähert sich bezüglich seiner Form mehr Nr. 4 mehr als Nr. 5 .

Die Ventrikularleisten stehen fast horizontal und sind $14,8 \mathrm{~mm}$ lang, die Teile hinter ihnen messen $4,3 \mathrm{~mm}$; die sagittale Länge des Cavum medium misst 19,1 mm.

Die Rima vestibuli erscheint auf dem Horizontalschnitt schmal oval mit hinterer Spitze; ihre Länge misst 18,4, ihre grösste Breite ist gleich $6,4 \mathrm{~mm}$.

Die Rima vestibuli ist langgestreckt, lanzettförmig, hinten etwas abgestumpft; sie ist sagittal 17,7 mm lang, an ihrer breitesten Stelle misst sie $4,1 \mathrm{~mm}$.

Der Epikrikoidwulst ist longitudinal länger, aber verschmälert.

Die Pyramide der Vorderseite hat die Gestalt wie bei Ausguss Nr. 4, ist aber etwas breiter.

Das Gesamtvolumen hat abgenommen.

Am Ausguss kommt die Wirkung der beteiligten Muskeln, die auf einer Verschmälerung des Cavum laryngis beruht, welche, soweit die M. aryt. transv. et obliqui in Frage kommen, auf den hinteren Umfang der Kehlkopfhöhle sich erstreckt, in anschaulicher Weise zum Ausdruck.

12. Verhalten des Ausgusses des durch Kontraktion der Mm. crico-thyreoidei (2), crico-aryt. posteriores (3) und crico-aryt. laterales (4) veränderten Cavum laryngis. (cf. Fig. 12).

Der Ausguss des Vestibulum laryngis erscheint vergrössert, die Vorderwand plan. 
Die Ventrikularleisten fallen leicht nach vorne $a b$, messen in sagittaler Länge $18,0 \mathrm{~mm}$, die Teile hinter ihnen $4,6 \mathrm{~mm}$. Der Mittelraum ist ausnehmend lang und misst 22,6 $\mathrm{mm}$.

Die Rima vestibuli ist zungenförmig gestaltet und mit $22,1 \mathrm{~mm}$ sagittaler Länge auffallend verlängert, ihre breiteste Stelle misst $9,8 \mathrm{~mm}$.

Die Rima glottidis hat die Gestalt eines fast regelmässigen gleichschenkeligen Dreieckes mit abgerundeten Ecken, die drei Seiten sind fast geradlinig. Die sagittale Länge ist ebenfalls sehr gross und misst $21,1 \mathrm{~mm}$, ihre breiteste Stelle entspricht der Grundlinie des Dreiecks mit $7,5 \mathrm{~mm}$.

Der Epikrikoidwulst ist deutlich ausgesprochen, jedoch verbreitert und abgeflacht.

Die Pyramide des Punctum centrale ist regelmässig gebaut, entspricht der Grösse von Nr. 3, ist aber etwas verschmälert.

Das Gesamtvolumen ist vermehrt.

Es zeigt dieser Ausguss, dass die gleichzeitige Wirkung der drei genannten Muskeln nötig ist, wenn der mittlere Kehlkopfraum sowie die Rimae glottidis und vestibuli die maximale Längenausdehnung erreichen sollen.

13. Verhalten des Ausgusses des durch Kontraktion der Mm. crico-thyreoidei (2), crico-aryt. posteriores (3) und arytaen. obliqui et transversi (5) veränderten Cavum laryngis. (cf. Fig. 13).

Der Ausguss des oberen Kehlkopfraumes zeigt eine zwischen Nr. 2 und 3 stehende Grösse, ist aber hinten etwas verschmälert.

Die Ventrikularleisten fallen wieder leicht wach vorn ab und sind sagittal 16,7 mm lang, die hinteren Teile 3,0 mm. Der Mittelraum misst $19,7 \mathrm{~mm}$ in sagittaler Länge.

Die Rima vestibuli ist im ganzen oval geformt, vorne spitzer zulaufend, am hinteren Ende mehr geradlinig begrenzt. Ihre sagittale Länge misst 18,8, ihre grösste Breite beträgt $8,4 \mathrm{~mm}$. 
Die Rima glottidis stellt ein vorn zugespitztes Oval dar. Ihre sagittale Länge ist gleich $18,0 \mathrm{~mm}$, ihre Breite 7,9 $\mathrm{mm}$.

Der Epikrikoidwulst ist stark ausgesprochen und ähnelt dem von Ausguss 5.

Die Pyramide entspricht etwa der von Ausguss 5, ist aber etwas höher.

Das Gesamtvolumen ist etwas vermehrt.

Dieser Ausguss steht seiner Gestalt nach am nächsten dem Ausguss 6. Seine vordere Hälfte zumal zeigt mit dem letzteren eine grössere Übereinstimmung, nur ist sie etwas schmäler; auch die hintere Hälfte erscheint verschmälert gegenüber Nr. 6, was auf Rechnung der Mm. arytaen. obliqui et transversi zu setzen ist, welche infolge des Zusammenrückens der Aryknorpel bei gleichzeitiger Kontraktion der $\mathrm{Mm}$. crico-thyreoidei und cricoaryt. posteriores auch auf die vordere Hälfte des Cavum laryngis etwas verengernd einwirken müssen. -

Vergleicht man den Ausguss mit Nr. 5, so erkennt man ferner, dass die $\mathrm{Mm}$. aryt. obliqui et transversi bei gleichzeitiger Kontraktion der Mm. crico-thyr. und crico-aryt. posteriores nicht so stark auf den hinteren Umfang des Cavum laryngis medium

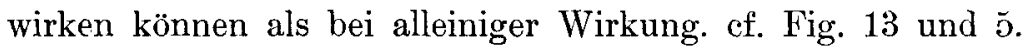

14. Verhalten des Ausgusses des durch Kontraktion der Mm. crico-thyreoidei (2), crico-arytaen. laterales (4) und arytaen. obliqui et transversi (5) veränderten Cavum laryngis. (cf. Fig. 14).

Der Ausguss des Vestibulum laryngis hat stark an Volumen abgenommen und läuft nach unten und hinten ungemein schmal zu.

Die Ventrjkularleisten stehen horizontal und messen in ihrer sagittalen Länge $16,9 \mathrm{~mm}$, in dem hinter ihnen gelegenen Teil 2,4 $\mathrm{mm}$. Der Mittelraum ist 19,3 $\mathrm{mm}$ lang.

Die Rima vestibuli hat die Gestalt des Durchschnittes durch eine schwache Konvexlinse, indem sie sowohl nach vorn als nach hinten sich gleichmässig verjüngt. Ihre sagittale Länge misst $18,1 \mathrm{~mm}$, ihre breiteste Stelle liegt fast in der Mitte und misst $3,6 \mathrm{~mm}$. 
Die Rima glottidis hat langgestreckte spindelförmige Gestalt, ist am hinteren Ende kaum verbreitert und zugespitzt. Ihre sagittale Länge ist gleich $19,5 \mathrm{~mm}$, ihre grösste Breite liegt weit nach hinten und beträgt $2,4 \mathrm{~mm}$.

Der Epikrikoidwulst ist ausnehmend stark und grösser als bei sämtlichen anderen Ausgüssen. Desgleichen ist die Einsenkung an der Vorderwand des Cavum inferius vorhanden, wie sie bereits bei Nr. 7 beschrieben wurde.

Die Pyramide des Punctum centrale ist sehr schmal und in die Länge gezogen.

Das Gesamtvolumen des Ausgusses ist stark vermindert.

Der Ausguss gleicht am meisten Nr. 7. Dadurch, dass zu den bei Nr. 7 in Betracht kommenden Muskeln noch die Wirkung der M. aryt. obliq. et transvers. hinzutritt, kommt es zu einer intensiveren Verschmälerung des hinteren 'Teiles der Kehl. kopfhöhle, welche nicht nur den oberen, sondern auch den mittleren Larynxraum betrifft. Bei dieser Muskelkombination ist die Länge der Stimmritze die grösstmögliche; es ist merkwürdigerweise die Länge der Rima glottidis hier sogar bedeutender als die der Rima vestibuli. Dass die Breite der Rima glottidis in ihrem mittleren Abschnitt bei Nr. 7 noch geringer ist als bei 14, erklärt sich dadurch, dass die Mm. arytaen. obliq. et transv. den Mm. crico-aryt. laterales etwas entgegenarbeiten und dem. nach die Processus vocales sich nicht so nahe kommen als wie es 7 zeigt, bei welchem Ausguss die Wirkung der Mm. aryt. obliq. et transv. ausgeschlossen ist. -- - -

Sehr lehrreich scheint mir auch der Vergleich von Ausguss 7 und 14 in Bezug auf die Frage zu sein, welche Muskeln zusammenhelfen müssen, wenn ein vollständiger Schluss der Rima glottidis zustande kommen soll. Ein solcher wurde bei meinen Versuchen weder durch die kombinierte Aktion der $\mathrm{Mm}$. crico-thyr. und laterales erreicht, noch auch dadurch zustande gebracht, dass der Wirkung der zwei genannten Muskeln noch 
die der Mm. arytaen. obliq. et transvers. hinzugefügt wurde. Somit lässt sich per exclusionem folgern, dass zum vollständigen Schluss der Rima glottidis noch die Kontraktion der Mm. vocales hinzukommen muss. - Um über diese Frage nun völlige Klarheit zu schaffen, habe ich an einem Kehlkopf neben der Wirkung der drei genannten Muskeln auch noch die der beider. seitigen Mm. vocales nachzuahmen gesucht. Dies geschah dadurch, dass zunächst zwei mit Knoten versehene Fäden durch den unteren Teil je eines Aryknorpels in der Höhe des Processus vocalis in sagittaler Richtung nach vorne durchgezogen wurden, die dann weiter in je einem Ligamentum vocale verlaufend den vorderen Ansatz des Stimmbandes und den Schildknorpel durchbohrten und an dessen Vorderfläche zum Vorschein kamen. Jedoch durften die Fäden nicht $\mathrm{zu}$ nahe dem freien Rande der Stimmbänder durchgezogen sein, sondern etwa $1 \mathrm{~mm}$ davon entfernt. Durch Anziehen der beiden Fäden wurde der Processus vocalis samt Aryknorpel etwas nach vorne bewegt. In gleicher Weise wurden in entgegengesetzter Richtung zwei Fäden durch den Kehlkopf geführt, deren vordere Enden mit Knoten versehen wurden und an denen von rückwärts her ein Zug ausgeübt wurde.

Durch Anziehen der vorderen und hinteren aus den Knorpeln herausführenden Fäden mussten die Ansatzstellen der Stimmbänder einander genähert werden, wodurch die Kontraktion der $\mathrm{Mm}$. vocales am natürlichsten zum Ausdruck gebracht wurde.

Der Ausfall dieses Versuches bestätigte die Voraussetzung, indem in der erörterten Weise die Stimmritze völlig geschlossen werden kann.

Es bedarf keiner weiteren Ausführung, dass sich die von mir hergestellten Kehlkopfausgüsse vorzüglich dazu eignen, die Korrelationen, welche zwischen den einzelnen Muskeln hinsichtlich ihres Einflusses auf das Kehlkopfinnere bestehen, eingehender zu studieren. Da ich die Ausgüsse von zwei Seiten her 
bildlich zur Darstellung gebracht habe, ferner in die Figuren 1-14 die Lumina des Kehlkopfes abgebildet habe, wie sie sich auf Sagittal-, Frontal- nnd Horizontal-Schnittebenen darstellen, so ist das Studium dieser Verhältnisse dem Leser wesentlich erleichtert und ich sehe deshalb davon ab, an dieser Stelle eine eingehendere Erörterung derselben zu geben, zumal ich ja dabei vieles wiederholen müsste, was bereits früher betont worden ist.

Ich beschränke mich daher darauf, zum Schlusse die wesentlichsten Ergebnisse meiner Untersuchungen über die Wirkung der Kehlkopfmuskeln auf Gestalt und Ausdehnung des Cavum laryngis zusammenzufassen:

1. Die grösste Volumverminderung der Kehlkopflıöhle wird durch gleichzeitige Kontraktion der Mm. crico-thyreoidei und crico-arytaenoidei laterales bedingt, wenn hierzu noch die Kontraktion des $\mathrm{Mm}$. arytaen. transversi et obliqui tritt.

2. Die grösste Volumvermehrung der Kehlkopfhöhle kommt bei alleiniger Wirkung der $\mathrm{Mm}$. crico-arytaenoidei posteriores zustande.

3. Die grösste Verengerung der Rima glottidis tritt bei gleichzeitiger Kontraktion der Mm. crico-thyreoidei und cricoaryt. laterales ein.

4. Der vollständige Schluss der Rima glottidis kommt zustande, wenn gleichzeitig die Mm. crico-thyreoidei, crico-arytaen. laterales, arytaenoidei transversi et obliqui und noch die $\mathrm{Mm}$. vocales kontrahiert sind.

5. Die grösste Erweiterung der Rima glottidis findet sich bei gleichzeitiger Wirkung der $\mathbf{M m}$. crico-arytaen. posteriores und arytaenoidei transversi et obliqui.

6. Die grösste (sagitt.) Verlängerung der Rima glottidis (und vestibuli) tritt bei gleichzeitiger Kontraktion der $\mathrm{Mm}$. cricothyreoidei, crico-arytaenoidei posteriores und crico-aryt. laterales ein. 
7. Die grösste (sagitt.) Verkürzung der Rima glottidis kommt bei alleiniger Kontraktion der $\mathrm{Mm}$. crico-arytaen. posteriores zustande.

Tabelle der Maasse (in Millimetern).

\begin{tabular}{|c|c|c|c|c|c|}
\hline $\begin{array}{c}\mathrm{Nr} . \\
\text { des Kehl- }\end{array}$ & \begin{tabular}{|l|} 
Sagittale \\
Länge des
\end{tabular} & Linge (sa & der Rima & Breiteste ( & l) Stelle der \\
\hline kopfs & Cavum medium. & Vestibuli & glottidis & vestibuli & glottidis \\
\hline 1 & 17,4 & 17,0 & 16,7 & 8,0 & 5,5 \\
\hline 2 & 19,2 & 18,8 & 18,0 & 6,0 & 3,5 \\
\hline 3 & 17,6 & 17,4 & 16,4 & 11,2 & 9,6 \\
\hline 4 & 18,1 & 17,6 & 17,3 & 5,6 & 3,9 \\
\hline 5 & 17,0 & 16,5 & 16,5 & 7,9 & 7,1 \\
\hline 6 & 18,6 & 18,2 & 17,5 & 9,5 & 8,9 \\
\hline 7 & 21,3 & 20,5 & 19,3 & 4,2 & 2,2 \\
\hline 8 & 18,6 & 18,1 & 17,5 & 6,6 & 3,9 \\
\hline 9 & 20,5 & 19,9 & 19,1 & 10,8 & 9,1 \\
\hline 10 & 16,8 & 16,1 & 17,0 & 11,1 & 10,5 \\
\hline 11 & 19,1 & 18,4 & 17,7 & 6,4 & 4,1 \\
\hline 12 & 22,6 & 22,1 & 21,1 & 9,8 & 7,5 \\
\hline 13 & 19,7 & 18,8 & 18,0 & 8,4 & 7,9 \\
\hline 14 & 19,3 & 18,1 & 19,5 & 3,6 & 2,4 \\
\hline
\end{tabular}

An dieser Stelle möge es mir gestattet sein, meinem sehr verehrten Lehrer, Herrn Professor Dr. L. Gerlach für die Anregung zu den interessanten Untersuchungen und für seine reichliche Unterstützung bei allen Teilen meiner Arbeit, meinen verbindlichsten Dank abzustatten.

Zu Dank verpflichtet bin ich auch Herm Professor Hauser für die freundliche Überlassung des Kehlkopfmaterials. 


\title{
Erklärung der Tafeln.
}

\author{
Tafel XXX/XXXI.
}

Seitenansicht der Kehlkopfausgüsse.

Fig. 1. Neutralstellung.

Fig. 2. Wirkung d. M. crico-thyreoidei.

Fig. 3. Wirkung d. M. crico-aryt. posteriores.

Fig. 4. Wirkung d. M. crico-aryt. laterales.

Fig. 5. Wirkung d. M. arytaen, transv. et obliq.

Fig. 6. Komb. von 2 und 3.

Fig. 7. Komb. von 2 und 4.

Fig. 8. Komb. von 2 und 5 .

Fig. 9. Komb. von 3 und 4 .

Fig. 10. Komb, von 3 und 5 .

Fig. 11. Komb. von 4 und 5 .

Fig. 12. Komb, von 2, 3 und 4.

Fig. 13. Komb. von 2, 3 und 5.

Fig. 14. Komb. von 2, 4 und 5 .

Diese Figg. entsprechen auch denen der anderen Tafeln.

\section{Tafel XXXII/XXXIII.}

Vorderansicht der Ausgüsse.

\section{Tafel XXXIV und XXXV.}

Reihe a: Sagittalschnitte der Ausgüsse.

Reihe b: Frontalschnitte der Ausgüsse.

Reihe c: Horizontalschnitt der Rima vestibuli.

Reihe d: Horizontalschnitt der Rima glottidis.

Alles Übrige ergiebt sich aus dem Text. 